Revista de
Economild
Contemporâned

\title{
O DEBATE ENVOLVENDO O EFEITO ACELERADOR NA CONTROVÉRSIA SOBRE O MODELO DO SUPERMULTIPLICADOR SRAFFIANO
}

\author{
Vivian Garrido Moreira ${ }^{a}$ \\ Franklin Serrano ${ }^{b}$

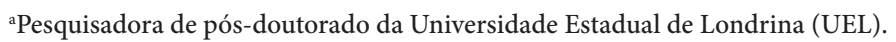 \\ Londrina, PR, Brasil. ORCID: http://0000-0002-3326-4737. \\ 'Professor associado do Instituto de Economia da Universidade Federal do Rio de Janeiro. \\ Rio de Janeiro, RJ, Brasil. ORCID: http://0000-0002-9981-9029.
}

Artigo recebido em 20/03/2018 e aceito para publicação em 08/04/2019.

RESUMO: Este trabalho discute os requisitos teóricos para o ajustamento da capacidade produtiva à tendência da demanda, no contexto do modelo do supermultiplicador sraffiano, por meio de sua controvérsia na literatura, que girou basicamente em torno da hipótese de acelerador rígido. Para alguns isso implicaria em contínua plena utilização da capacidade, requerendo que a demanda se ajustasse à capacidade. Para outros, implicaria em excessiva sensibilidade do investimento à demanda, gerando instabilidade. Desenvolvimentos recentes, no entanto, confirmam que não há suposição de acelerador rígido, sendo o modelo plenamente compatível com o acelerador flexível e atendendo às condições de crescimento liderado pela demanda.

PALAVRAS-CHAVE: demanda efetiva; acelerador; supermultiplicador.

CLASSIFICAÇÃO JEL: B51; E11; O41. 


\title{
THE DEBATE ON THE ACCELERATOR EFFECT IN THE CONTROVERSY ABOUT THE SRAFFIAN SUPERMULTIPLIER MODEL
}

\begin{abstract}
This paper discusses the theoretical requirements for the adjustment of productive capacity to demand, in the context of the controversy about the Sraffian supermultiplier growth model, that revolved basically around the assumption of a rigid accelerator. To some, this assumption would imply in the continuous full utilization of capacity, requiring demand to adjust to capacity. To others, it implied excessive sensitivity of investment to demand and instability. Recent developments, however, confirm that no assumption of a rigid accelerator is made, the model being fully compatible with a flexible accelerator and all the conditions required for demand led growth.
\end{abstract}

KEYWORDS: effective demand; accelerator; supermultiplier. 


\section{INTRODUÇÃO}

O objetivo da pesquisa que levou ao modelo de crescimento do supermultiplicador sraffiano proposto em Serrano (1995a e 1995b) era mostrar como e sob que condições a evolução da tendência da demanda efetiva poderia determinar, num prazo mais longo, os níveis e a tendência de crescimento do estoque de capital e do próprio produto potencial da economia, por meio do ajustamento da capacidade produtiva à tendência da demanda.

No entanto, a análise teórica incompleta do comportamento do modelo durante tal processo de ajustamento gerou ambiguidades e questionamentos teórico-metodológicos no debate que se seguiu à publicação de Serrano (1995a). Na medida em que esse debate, após alguns anos, tem sido novamente colocado em pauta e gerado discussões muito recentes, julgamos oportuno trazer à tona os principais integrantes nele envolvidos. ${ }^{1}$ As críticas basicamente oscilaram entre dois pontos, frequentemente utilizados de forma combinada: um de que o modelo reproduz de alguma forma a lei de Say, logo, seria liderado pela oferta e não pela demanda; outro, de que, quando liderado pela demanda, dependeria de condições muito particulares e improváveis, envolvendo a hipótese de acelerador rígido, que, aliás, é, ela própria, objeto de controvérsia.

No presente trabalho vamos resenhar criticamente a discussão teórica envolvida nesse debate à luz de resultados de pesquisas mais recentes. $\mathrm{O}$ artigo está organizado da seguinte forma: inicialmente apresentamos o conceito de acelerador rígido e sua relação com a tecnologia e o grau de utilização da capacidade produtiva (seção 2). A seguir, apresentamos brevemente o modelo do supermultiplicador sraffiano em sua formulação original de 1995 (seção 3). Passamos então às críticas a essa formulação (seção 4), iniciando com as críticas conceituais e formais propostas por Trezzini, Palumbo, Park e Barbosa Filho. Mostraremos que tais críticas envolvem uma interpretação inadequada tanto do supermultiplicador sraffiano quanto do próprio conceito de acelerador rígido. Na sequência, discutimos as críticas e alternativas propostas por Schefold, De-Juan e White, que partem de uma interpretação adequada do acelerador rígido, porém não se aplicam ao supermultiplicador sraffiano.

1 O interesse na literatura sobre o fechamento teórico proposto pelo supermultiplicador sraffiano foi renovado a partir da sua redescoberta e adoção por autores neo-kaleckianos (LAVOIE, 2014; ALLAIN, 2014; LAVOIE, 2016). No campo sraffiano houve a publicação do simpósio "Pierangelo Garegnani, the Classical Surplus Approach and Demand-led Growth” (CESARATTO, 2015), em que foi publicada uma análise completa das condições de estabilidade dinâmica do modelo (FREITAS e SERRANO, 2015) e também (postumamente) o trabalho seminal de Garegnani (1962[2015]), que iniciou o programa de pesquisa sraffiano sobre o ajustamento da capacidade à demanda (CESARATTO, 2015; GARRIDO MOREIRA e SERRANO, 2018). 
Finalmente, apresentamos o supermultiplicador sraffiano em sua formulação completa, com acelerador flexível (seção 5), e fechamos o artigo com breves observações finais (seção 6).

\section{A TAXA DE INVESTIMENTO REQUERIDA NO CONTEXTO DO ACELERADOR RÍGIDO}

O chamado efeito acelerador em sua versão mais simples ou rígida é deduzido para uma dada relação técnica capital-produto potencial, $v=K / Y^{*}$, onde, em geral, supõe-se que o produto potencial se refere a um nível normal ou desejado de utilização da capacidade e que, portanto, pressupõe a existência de alguma ociosidade planejada, $\alpha$, de tal forma que a relação técnica entre o capital e o máximo que poderia ser produzido seria dada por $v_{\max }=v(1+\alpha)$. Assim, o estoque de capital disponível no início do período (ou seja, $K=K_{-1}+I_{-1}$, onde $I$ é o investimento líquido e o subscrito $(-1)$ denota o período discreto anterior) obtido a partir da utilização da capacidade produtiva ao nível normal seria dado por:

$$
K=v Y^{*}
$$

E o investimento líquido requerido para um aumento do produto entre o período atual e o próximo, mantendo normal o grau de utilização da capacidade, seria dado por:

$$
I=v\left(Y_{+1}^{*}-Y^{*}\right)
$$

As relações acima implicam que existe uma relação técnica necessária entre a taxa de investimento e a taxa de crescimento do produto, partindo-se de uma posição inicial de utilização normal, pois uma maior parcela do produto potencial terá de ser devotada ao investimento caso seja desejada uma maior taxa de crescimento do produto potencial no período seguinte. Essa taxa de investimento será dada por:

$$
I / Y^{*}=v \frac{\left(Y_{+1}^{*}-Y^{*}\right)}{Y^{*}}=v g_{+1}^{*}
$$

Vamos chamar essa relação entre taxa de investimento e taxa de crescimento de taxa de investimento requerida. Note que se, no período corrente, o grau efetivo da utilização da capacidade for diferente do normal (isto é, se $u=Y / Y^{*}$ for diferente de 1), a equação (3) não implica uma relação necessária entre taxa de investimento sobre o produto efetivo e taxa de crescimento da capacidade produtiva no período seguinte. Formalmente, temos que: 


$$
\frac{I}{Y^{*}}=\frac{I}{Y} \frac{Y}{Y^{*}} \text { ou } \frac{I}{Y^{*}}=\frac{I}{Y} u
$$

Logo,

$$
v g_{+1}^{*}=\frac{I}{Y} u \text { ou } g_{+1}^{*}=\frac{I / Y}{v} u
$$

mostra que a taxa de crescimento do estoque de capital e a taxa da capacidade produtiva dependem tanto da taxa de investimento sobre o produto corrente quanto do grau efetivo de utilização da capacidade. Uma vez que o que realmente importa para determinar a taxa de crescimento da capacidade produtiva é o montante de investimento, sobre o produto potencial e não a razão entre investimento e o produto corrente (taxa de investimento), quanto menor for o grau de utilização de capacidade, menor será a taxa de crescimento da capacidade produtiva associada a uma certa taxa de investimento $I / Y$. Da mesma forma, se o grau efetivo de utilização da capacidade for maior que o normal, teremos uma taxa de crescimento do produto potencial mais alta para uma dada taxa de investimento. Por outro lado, é importante ressaltar que uma equação semelhante a (3) inevitavelmente aparecerá em qualquer modelo de crescimento em que o grau de utilização da capacidade tender a seu nível normal, $(u=1)$, seja qual for a natureza do mecanismo que leva a essa tendência (a demanda se ajustando à capacidade produtiva ou a própria capacidade se ajustando à demanda). Em um modelo de crescimento liderado pela demanda, a taxa de investimento induzido (que chamaremos também, como na literatura do supermultiplicador, de propensão marginal a investir), no caso em que se faz a hipótese de acelerador rígido, é determinada pela taxa de investimento requerida por uma dada taxa de crescimento esperada da demanda, $\left(g^{e}\right)$. Esta última, por sua vez, é determinada pela taxa efetiva de crescimento da demanda do período imediatamente anterior, $\left(g_{-1}\right)$, a partir de uma versão "ingênua” de expectativas adaptativas:

$$
I / Y=v g^{e}=v g_{-1}
$$

Essa seria a formulação básica da taxa de investimento do acelerador rígido (em tempo discreto). Mas a função de investimento do acelerador rígido não implica, por si só, que o grau de utilização da capacidade seja igual ao normal em nenhum período. $\mathrm{O}$ que o acelerador rígido postula é que os investidores tentam investir de forma a ajustar todo o estoque de capital às mudanças da demanda esperada em cada período. Inclusive, exatamente por conta dessa reação exagerada, a função investimento do acelerador rígido em geral leva a maiores flutuações do grau de utilização efetivo da capacidade. 


\section{O SUPERMULTIPLICADOR SRAFFIANO² EM SUA FORMULAÇÃO ORIGINAL}

O ponto central do modelo do supermultiplicador sraffiano é a existência, no contexto do processo de acumulação de capital, de uma tendência ao ajustamento da capacidade produtiva à demanda. Nessa visão existem três condições teóricas gerais necessárias para a ocorrência desse ajustamento:

(i) Investimento induzido: o montante agregado do investimento que cria capacidade produtiva para o setor privado deve ser endogenamente determinado e seguir o princípio de ajuste do estoque de capital para que, num prazo mais longo, tenda ao valor requerido para atender à demanda efetiva agregada e sua evolução no tempo.

(ii) Gastos autônomos (isto é, não induzidos pelo produto) que não criam capacidade produtiva para o setor privado: sua existência permite que, ao longo do processo de ajustamento, o investimento (e posteriormente a capacidade produtiva) cresça mais rápido ou menos rápido que o consumo (ou os gastos não criadores de capacidade, em termos mais gerais), tornando o ajustamento da capacidade à demanda, logicamente possível. Essa condição evita o problema da instabilidade harrodiana comum em modelos em que o consumo é todo induzido, fazendo com que variações no nível agregado de investimento sempre gerem variações exatamente proporcionais no consumo, impedindo o ajustamento endógeno da taxa de investimento. ${ }^{3}$

(iii) Propensão marginal a gastar menor do que 1: isso requer que o nível e a taxa de crescimento dos gastos autônomos não gerem investimento e consumo induzidos cronicamente excessivos, que tornem inviável o próprio regime de crescimento liderado pela demanda, mesmo que o modelo tenha gastos autônomos. Se acaso os gastos induzidos pelo produto se tornarem maiores que $100 \%$ do próprio produto, a produção nunca conseguiria se ajustar à demanda.

No supermultiplicador sraffiano, o investimento privado que gera capacidade produtiva é totalmente induzido por meio de alguma versão do acelerador (atendendo à primeira condição acima) na qual a taxa de investimento líquido reage à taxa de

\footnotetext{
2 O modelo foi desenvolvido independentemente por Bortis (1979) e Serrano (1995b) e foi chamado de supermultiplicador por ambos, pela semelhança formal com o modelo de Hicks (1950), apesar dos diferentes propósitos e hipóteses. Todavia, Cesaratto (2017) descobriu que, muito antes, Ackley (1963), num modelo econométrico para a Itália, adaptou o supermultiplicador de Hicks a uma análise de crescimento liderado pela demanda autônoma.

3 Sobre esse tema, ver Serrano, Freitas e Bhering (2018).
} 
crescimento esperada da demanda, $\left(g^{e}\right)$, fazendo o investimento e a posteriori o estoque de capital tenderem a crescer mais rápido do que o crescimento da demanda total corrente $(g)$ quando $g^{e}$ aumenta:

$$
\frac{I}{Y}=v g^{e}
$$

Numa versão bem simples, omitimos o governo e o setor externo, supomos que a distribuição de renda é determinada a partir de um salário real exógeno, que a propensão marginal a poupar dos lucros é igual a $1 \mathrm{e}$, adicionalmente, que todo o consumo dos capitalistas é autônomo. ${ }^{4}$ Assim, a parcela dos salários na renda, conjuntamente com os hábitos de consumo dos trabalhadores, determina a propensão marginal a consumir da economia (ou seja, $c=c_{w} w$, onde $c_{w}$ é a propensão marginal a consumir dos salários e $w$ é a parcela dos salários na renda). O modelo basicamente consiste, então, da combinação de uma função consumo que contenha um componente de consumo autônomo $(Z)$ que cresce a uma taxa exógena $(z)$ (o que atende à segunda condição necessária descrita acima) e uma função de investimento induzido. Qualquer aumento no nível de investimento eleva o produto e faz com que a razão $(Z / Y)$ se reduza, aumentando, então, a taxa de investimento. A queda de $(Z / Y)$, por sua vez, corresponde a um aumento da parcela poupada da renda:

$$
\frac{I}{Y}=\frac{S}{Y}=\left(s-\frac{Z}{Y}\right)
$$

Essa endogeneidade da taxa de investimento a partir de variações do nível de investimento é que torna possível que a capacidade possa crescer mais (menos) que a demanda e eventualmente eliminar a sobreutilização (subutilização) da capacidade. O nível de atividade na posição de longo prazo será dado pelos produtos entre os gastos autônomos e o supermultiplicador, em que o último se refere à recíproca de um menos a propensão marginal a gastar (soma da propensões marginais a consumir $c$ e investir $\frac{I}{Y}$ ):

$$
Y=\frac{Z}{\left(1-c-v g^{e}\right)}
$$

No entanto, não é possível garantir que o grau efetivo de utilização da capacidade verificado na posição descrita em (9) seja necessariamente normal e que a taxa esperada

4 Esta hipótese não é necessária para os resultados e o modelo pode conter um componente de consumo induzido dos lucros (SERRANO, 1995a). 
seja igual à taxa efetiva de crescimento da demanda. O que Serrano (1995a) faz, sem apresentar uma análise formal do processo, é supor que, de alguma maneira, a revisão das expectativas dos investidores fará com que a economia tenda a convergir lentamente para uma posição de pleno ajustamento, na qual o grau de utilização é normal, e a demanda, o produto e o nível da própria capacidade produtiva $\left(Y^{*}\right)$ cresçam liderados pela expansão dos gastos autônomos. A ausência de uma análise formal explícita do ajuste de expectativas é a provável fonte dos equívocos interpretativos que veremos na sequência. Por outro lado, o supermultiplicador sraffiano, quando da sua concepção, concentrou-se numa modificação da função consumo e não no desenvolvimento de uma função investimento. Nesse sentido, a inclusão de algum gasto autônomo que não cria capacidade no modelo, embora não seja suficiente, é estritamente necessária para garantir que a "ordem" do ajuste seja sempre da capacidade para a demanda, bastando, para isso, qualquer função investimento que seja compatível com o princípio de ajuste do estoque de capital. ${ }^{5}$ Mas, por algum motivo, o debate se desviou basicamente para a formação de expectativas ao longo do ajuste ou para qual seria a forma específica da função investimento. Não é imediato, como não costuma ser na ciência, o porquê de ter sido essa e não aquela a trilha percorrida pelo desenvolvimento das ideias. Mas, nesse caso, o desvio da questão central parece ter fomentado uma significativa multiplicação de ideias confusas sobre o tema.

Voltando, portanto, à hipótese simplificada da versão inicial do modelo de que as expectativas sejam revistas na dimensão necessária e suficiente para permitir um processo estável de ajustamento, a taxa de crescimento esperado da tendência da demanda $\left(g^{e}\right)$ converge para " $z$ ". Essa convergência faria a taxa de investimento se aproximar da taxa requerida. A expressão (9) tenderia, então, a se transformar em (10), a posição de ajustamento "secular" (ver nota número 7):

$$
Y^{*}=Y=\frac{Z}{(1-c-v z)}
$$

\footnotetext{
Qualquer função investimento compatível com o princípio de ajuste do estoque de capital (MATTHEWS, 1959) é compatível com a existência conceitual e empírica de decisões individuais de investimento autônomo. O que a operação desse princípio aponta é que, no agregado e num prazo mais longo, há uma tendência de que os efeitos dos investimentos autônomos tanto na demanda quanto na capacidade produtiva da economia sejam compensados posteriormente por mudanças na direção oposta nos investimentos induzidos. A justificativa completa encontra-se em Cesaratto et al. (2003).
} 


\section{O ACELERADOR RÍGIDO E O SUPERMULTIPLICADOR REINTERPRETADOS PELOS CRÍTICOS}

Na medida em que as críticas a Serrano (1995a) possuem uma base muito semelhante, faz-se mister um esforço de compreensão do que estava presente nas versões originais destas, capaz de despertar tal interpretação comum, que veremos ser equivocada e oposta à concepção teórica do supermultiplicador sraffiano. ${ }^{6}$

Um ponto aparentemente ingênuo, mas que provavelmente é relevante, tem a ver com a apresentação da "forma final" do modelo: a posição de pleno-ajustamento de longo prazo $^{7}$ apresentada na equação (10). Ressalte-se que esta configura um centro de gravitação para a economia, num prazo secular, quando realizado determinado ajuste da capacidade a uma determinada variação da demanda agregada. Os detalhes desse ajuste, que será gradual e estável, serão tratados na seção 5, no contexto do acelerador flexível. Nessa posição abstrata de convergência e de tendência, que não necessariamente precisa ocorrer com precisão, há um equilíbrio, $(i)$, no qual o grau de utilização é normal, (ii), e a taxa de crescimento econômico é igual à taxa de crescimento dos gastos autônomos. Além desses dois, ainda poderíamos acrescentar mais um elemento importante para a discussão: não se considera a participação de investimentos autônomos criadores de capacidade ${ }^{8}$ entre os componentes de demanda, (iii). Uma visão isolada desses três elementos, sem considerar o contexto teórico do modelo em questão, pode levar à conclusão precipitada de que estes seriam antagônicos com o princípio da demanda efetiva.

Barbosa-Filho (2000) por exemplo, refere-se à posição em (10) como "uma relação quantitativa estática”. Esta poderia ser tipicamente uma observação aplicável a uma visão do resultado que ignora o processo. A partir de considerações desse tipo, os críticos, resumidamente, realizam uma das seguintes leituras do supermultiplicador sraffiano:

Leitura 1: tratar-se-ia de um modelo no qual o crescimento não tem como ser liderado pela demanda;

Leitura 2: tratar-se-ia de um modelo que, se for liderado pela demanda, só vale com acelerador rígido;

6 Para uma revisão dessas críticas, ver Garrido Moreira (2007).

7 O horizonte temporal de longo prazo do supermultiplicador sraffiano não é o mesmo de vários autores sraffianos. Serrano (1995a) menciona a expressão "prazo secular", mais longo que o comumente utilizado na literatura. Ver também Garrido Moreira e Serrano (2018). Para evitar essa confusão, Freitas e Serrano (2015) chamam de posição de longo prazo apenas a equação (9), enquanto (10) é chamada de posição plenamente ajustada.

8 Ver nota número 5 acima. 
Leitura 3: tratar-se-ia de um modelo liderado pela oferta "e com acelerador rígido";

Leitura 4: tratar-se-ia de um modelo liderado pela demanda, mas que, como pressupõe um acelerador rígido, muito provavelmente não será dinamicamente estável.

Queremos mostrar que nenhuma dessas leituras está correta, não apenas devido a uma inadequação dos conceitos utilizados para analisar o supermultiplicador, mas também devido a um entendimento equivocado dos próprios conceitos, principalmente aqueles que dizem respeito às características e aos efeitos de um acelerador rígido.

\subsection{LEITURAS (1) E (2): CRESCIMENTO LIDERADO PELA DEMANDA OU PELA OFERTA? (TREZZINI E PALUMBO)}

As críticas de Trezzini (1995, ${ }^{9}$ 1998) e Palumbo e Trezzini (2003) ao supermultiplicador sraffiano se originam da sua tentativa de generalizar a argumentação de Garegnani (1992) para um contexto em que existem gastos autônomos que não criam capacidade, procurando mostrar que o supermultiplicador sraffiano (que contém esse tipo de gasto) estaria sujeito às críticas de Garegnani a modelos que mantêm o grau de utilização da capacidade produtiva continuamente igual ao seu nível normal ou planejado.

No contexto da abordagem sraffiana, Garegnani (1992) argumenta que, se a economia opera sob o princípio da demanda efetiva, então o grau de utilização efetivo da capacidade em algum momento deve se desviar do seu nível normal, seja na posição inicial da economia ou então em algum período posterior - quando um nível de investimento menor (maior) do que a poupança potencial necessariamente irá levar a uma criação menor (maior) de capacidade no período subsequente independentemente da demanda que poderá vir a ocorrer em tal período. Dessa forma, segundo Garegnani (1992), não se deve esperar que se observe o grau de utilização efetivo igual ao normal ao longo do tempo, nem continuamente, nem em

9 Curiosamente, Trezzini (1995) não cita Serrano (1995a), apesar de os dois artigos terem sido publicados no mesmo número de uma revista e de Serrano (1995a, 1995b) citarem Trezzini (1995). Trezzini (1995) argumenta que suas críticas diriam respeito ao próprio modelo de Hicks (1950). Como Trezzini (1995) não apresenta nenhuma evidência textual e dado que Hicks jamais utilizou em qualquer texto seu modelo de ciclo do supermultiplicador para justificar a ideia de crescimento liderado pela demanda autônoma e uma tendência do ajustamento da capacidade à demanda (o que se oporia radicalmente à sua visão de que o crescimento de longo prazo depende da oferta), as críticas do artigo de Trezzini (1995) só fazem sentido se entendidas como endereçadas ao supermultiplicador sraffiano. 
média (média essa que, ao incluir a capacidade produtiva inicial, que é herdada do passado, não tem como incorporar o ajustamento que lhe sucede).

Baseado nessas ideias gerais, Trezzini $(1995,1998)$ segue apontando que a única taxa de crescimento da demanda capaz de permitir a contínua normalidade do grau de utilização seria a taxa garantida harrodiana. Uma vez que não haja nenhuma razão para que a demanda agregada cresça à taxa garantida, não há motivo para que o grau de utilização fique normal. Taxas de crescimento diferentes da garantida determinam um grau de utilização médio diferente do normal (a menos de alguma coincidência) mesmo que as firmas possuam perfeita previsão da demanda futura, pois a capacidade produtiva é determinada exogenamente por eventos no passado. O autor segue dizendo que a validade desse argumento independe da presença de gastos autônomos no modelo. Trezzini apresenta as duas diferentes possibilidades de crescimento equilibrado, inicialmente sem a presença de gastos autônomos e depois os acrescentando, mostrando apenas uma redução da taxa garantida nesse último caso. Trezzini argumenta que, para efeito de análise de trajetórias plenamente ajustadas (que é como ele entende que seja o caso do supermultiplicador), requer que a taxa de crescimento da demanda se mantenha continuamente igual à taxa garantida, com ou sem gastos autônomos, e propõe que a inclusão dos gastos autônomos não alteraria o argumento central já presente em Garegnani (1992), e, portanto, não serviria como solução para estabilizar o processo de ajustamento da capacidade à demanda.

Não parece haver outra explicação para essa conclusão que não a incompreensão do que listamos como condição necessária n 2 na seção 3. Se não, vejamos.

Trezzini diz não encontrar justificativas sobre de que maneira - supondo, por exemplo, uma elevação na taxa de crescimento dos gastos autônomos - os investimentos induzidos poderiam ser calibrados no exato aumento necessário para chegar à posição de pleno-ajustamento, ou, ao menos, gravitar em torno dela, pois o caráter dual do investimento, que gera mais demanda logo que é realizado, mas só gera mais capacidade posteriormente, acarreta um desvio do grau de utilização em relação ao normal durante o processo de ajustamento. Mas, uma vez que a capacidade tenha atingido tamanho suficiente para operar o nível normal de utilização para o novo nível de demanda, as firmas deveriam então, segundo Trezzini, reduzir o nível absoluto de investimentos, não obstante o grau de utilização de fato estar ainda acima do normal. Porém, basta que poucas firmas não reduzam para que a demanda agregada continue implicando em sobreutilização da capacidade. Assim, o desvio do grau de utilização em relação à posição normal não se caracterizaria como um fenômeno de curto de prazo ou temporário, que poderia ser corrigido no longo prazo.

Toda essa argumentação seria muito razoável não fosse o fato de que é justamente a inclusão de gastos autônomos no modelo, o elemento que permite que o investimento 
cresça mais rápido que o consumo quando ajustamento é "para mais” ou que decresça mais rápido que o consumo quando o ajustamento é "para menos", tornando a capacidade cada vez menos "sobreutilizada" no primeiro caso ou "subutilizada" no segundo, sem que nenhum agente precise, em seu nível microeconômico, saber antecipar isso; basta apenas que continue agindo racionalmente, aumentando o investimento quando a capacidade estiver sobreutilizada e diminuindo no caso contrário.

Como Trezzini não reconhece a centralidade dos gastos autônomos para permitir esse ajustamento, entende que o supermultiplicador sraffiano só funciona se a taxa de crescimento da demanda autônoma (e todos os demais parâmetros do modelo) se mantiver constante por um período indefinidamente longo. Com isso, faz uma drástica reinterpretação do modelo, basicamente invertendo a causalidade e o sentido da equação (10). De acordo com essa nova interpretação: (i) a capacidade produtiva agora é exógena e corresponde à capacidade existente no período inicial; e (ii) a equação agora tem por objetivo definir qual é a taxa de crescimento esperada no futuro, $g_{w}$ (sendo esta, agora, a variável dependente da equação), que geraria investimento induzido e demanda suficiente no período corrente para garantir a plena utilização dessa capacidade inicial dada. A resposta a essa pergunta define uma variante da taxa garantida de Harrod, modificada pelo fato de que existem gastos autônomos que não criam capacidade:

$$
\begin{gathered}
Y^{*}=Y=\frac{Z}{\left(1-c-v g_{w}\right)} \\
g_{w}=\frac{(s-Z / Y)}{v}
\end{gathered}
$$

Acrescenta-se ainda mais uma hipótese: (iii) a economia deve se manter numa trajetória de steady state, onde a taxa de crescimento é constante, o que só ocorrerá se, por acaso, a taxa de crescimento dos gastos autônomos for exatamente igual a taxa garantida referida acima:

$$
z=\frac{(s-Z / Y)}{v}
$$

Se isso não ocorrer, ou $Z / Y$ aumentará no tempo e a própria taxa garantida será reduzida se $z>g_{w}$, ou $Z / Y$ irá diminuir no tempo e a taxa garantida tenderá a aumentar se $z<g_{w}$.

Não é fácil reconhecer nessa reinterpretação o supermultiplicador sraffiano discutido na seção 3. O que era um modelo de crescimento liderado pela demanda acaba, dessa forma, transformado num modelo de crescimento liderado pela oferta, onde a poupança potencial determina o nível de investimento. Essa transformação 
ocorre por conta da inversão de causalidade operada na passagem do supermultiplicador sraffiano para a equação (13) da taxa garantida modificada por gastos autônomos. Com essa reinterpretação, a variável dependente do modelo deixa de ser a pergunta central a que o supermultiplicador sraffiano tenta responder (com a equação (10)): qual o nível de capacidade produtiva que tende a ser criado para ajustar a capacidade a demanda? Já a questão totalmente diversa que Trezzini postula com sua taxa garantida modificada pelos gastos autônomos (equação (13)) é: a partir de um nível inicial dado exogenamente de capacidade produtiva, qual o nível e taxa de crescimento da demanda que permitiria a utilização normal da capacidade tanto no período inicial quanto indefinidamente nos períodos futuros? A própria pergunta de Trezzini implica num ajustamento da demanda à oferta, e, portanto, do investimento à poupança potencial.

Procurando explicar o porquê desse formato supostamente inadequado para um modelo de crescimento liderado pela demanda, Trezzini avança para a conexão com o acelerador rígido. Essa conexão se dá por meio de duas proposições, cada uma delas desencadeando uma linha de crítica. Ambas as linhas foram posteriormente utilizadas pelos críticos ao modelo. A primeira é de que o supermultiplicador sraffiano necessariamente suporia um acelerador rígido, já que, na posição plenamente ajustada, a propensão marginal a investir é igual a (já mencionada) taxa de investimento requerida, determinada pela relação técnica entre capital e produto potencial, $(v)$, e pela taxa de crescimento dos gastos autônomos, $(z)$. Até esse ponto não se questiona diretamente se o crescimento é liderado pela demanda. A segunda seria de que uma função de investimento induzido baseada no acelerador rígido seria incompatível com o princípio da demanda efetiva, pois implicaria na contínua plena utilização da capacidade. Aqui entra a associação direta do modelo com a crítica que lhe atribui a lei de Say. Conclui-se, enfim, que o modelo opera com contínua utilização normal da capacidade, inclusive na posição inicial da economia.

Sobre a primeira proposição, na realidade, qualquer função investimento induzido corretamente especificada que produzisse um modelo dinamicamente estável de crescimento liderado pela demanda levaria ao ajuste da capacidade e à tendência da propensão marginal a investir para a taxa de investimento requerida na posição de pleno ajustamento. Isso tanto poderia acontecer com o acelerador rígido quanto com o flexível, desde que o modelo seja estável. Entretanto, o supermultiplicador sraffiano, na verdade, não postula o acelerador rígido, e é plenamente compatível com o acelerador flexível. ${ }^{10}$

10 Versões do supermultiplicador sraffiano com hipóteses explícitas de acelerador flexível já se encontram em Serrano (2001), Cesaratto et al. (2003) e Serrano (2006), os dois primeiros citados em Palumbo e Trezzini (2003) e os dois últimos em Palumbo (2013). Apesar das citações, ambos mantêm o argumento de que o supermultiplicador sraffiano utiliza acelerador rígido. 
Nesse último caso, a função investimento não apenas é compatível, mas também torna mais provável que a propensão marginal a investir realmente tenda para o valor da taxa de investimento requerida. Aliás, o próprio Trezzini (1995) reconhece, numa nota de rodapé que passou desapercebida na literatura, não apenas que o acelerador flexível seria imune às suas críticas, mas também parece admitir o quão idiossincrática é a sua interpretação do acelerador rígido. Depois de dizer no texto que o acelerador rígido implica em contínuo plena utilização, na nota, Trezzini admite que:

This feature of the rigid accelerator has been corrected by the flexible formulations of the principle, which have been used exclusively, however, for the analysis of cyclical fluctuations. See Duesenberry (1958). Since our attention is focused on the long-run tendencies we shall not consider these flexible formulations. Neither shall we consider the ways in which the rigid accelerator has been actually used for the study of cyclical fluctuations without running into the logical problems described in the text, but progressively getting further away from the original behavioral hypotheses on which it was built. (TREZZINI, 1995, p. 7, fn 2; grifos nossos)

Sobre a segunda proposição, o acelerador rígido não é incompatível com desvios do grau de utilização da capacidade. Na realidade, conforme discutimos na seção 2, o acelerador rígido implica que as firmas desejam manter a capacidade plenamente ajustada à demanda esperada em cada período e, para isso, criam nova capacidade no montante exato do aumento da demanda esperada a cada período. Mas isso de forma alguma garante que as empresas serão capazes de realmente conseguir este resultado. Primeiro porque o modelo pode ser instável (o que vimos ser bastante provável) e, segundo porque, mesmo que o modelo seja estável, haverá flutuações inevitáveis no grau efetivo de utilização da capacidade, pelas defasagens entre demanda realizada, seu efeito na demanda esperada e o efeito capacidade dos investimentos. Dessa forma, mesmo um modelo de acelerador rígido estável garantiria apenas uma tendência ao grau de utilização normal e não que tal situação ocorresse continuamente ou nem mesmo numa média exata ao longo do tempo. Note que, mesmo que o modelo com acelerador rígido seja estável, durante a trajetória de ajustamento a choques exógenos, como mudanças nos parâmetros do modelo, o grau de utilização médio efetivo da capacidade não tem por que ficar igual ao normal, apenas tende a esse valor (MATTHEWS, 1959).

Há também o argumento (PALUMBO e TREZZINI, 2003) de que, mesmo que o supermultiplicador sraffiano não suponha utilização continuamente normal, mas apenas uma média igual à utilização normal, por um período longo, o modelo ainda seria incompatível com a independência da demanda em relação à oferta. Isso porque, 
nessa média, inclui-se também o período inicial, para o qual a capacidade produtiva instalada é, evidentemente, exógena. Para chegar a essa conclusão, os críticos que seguiram esse raciocínio incorporaram equivocadamente (pois indevidamente invertem a causalidade do modelo como vimos acima) nessa média o nível inicial exógeno da capacidade produtiva existente. Assim, mesmo que o processo que gera o nível endógeno de capacidade plenamente ajustada para a qual o modelo do supermultiplicador sraffiano tenderá se for estável, evidentemente isso não implica que o grau médio efetivo de utilização da capacidade será igual ao normal. Entretanto, Palumbo e Trezzini (2003) argumentam incorretamente que o supermultiplicador sraffiano supõe que a utilização é normal numa média exata de todos os períodos, inclusive o período inicial, em que a capacidade inicial é necessariamente exógena. Uma vez que a capacidade previamente existente foi determinada por decisões de investimento tomadas no passado, uma utilização média exatamente igual ao normal para o período todo, inclusive o período inicial, só poderia ser garantida se a própria demanda no período inicial se ajustasse à capacidade produtiva e não o oposto.

\subsection{LEITURA 3: LEI DE SAY COM ACELERADOR RÍGIDO? (BARBOSA-FILHO E PARK)}

A reinterpretação radical e invertida do supermultiplicador sraffiano proposta por Trezzini (e também Palumbo) influenciou outros autores em suas análises e críticas formais do modelo. Tais críticas se utilizaram dos elementos elencados na seção anterior, seja utilizando-os diretamente, seja combinando-os. Trezzini e Palumbo, porém, não procedem declaradamente uma crítica combinada, isto é, questionam o supermultiplicador como sujeito a uma das duas linhas críticas (correspondentes a duas proposições diferentes) mostradas na seção anterior: ou lei de Say, ou crescimento liderado pela demanda com acelerador rígido de um modo extremamente particular (desconsiderando qualquer outro modo para sua ocorrência), na qual a reação do estoque de capital às oscilações de demanda (por definição sempre completa) mantém continuamente o grau de utilização normal, o que pressupõe alguma forma de previsão perfeita de demanda. Assim, a literatura que repõe essas críticas eventualmente também as combina, gerando análises que, ao que tudo indica, apresentam uma leitura de um suposto supermultiplicador com lei de Say e acelerador rígido ao mesmo tempo. Uma notável contradição teórica, já que nenhum modelo pode ser liderado pela oferta e pela demanda ao mesmo tempo.

Barbosa-Filho (2000) argumenta que o supermultiplicador sraffiano, ao ser (supostamente) baseado na hipótese de acelerador rígido, seria inerentemente instável. Porém, o que o autor chama de instabilidade não diz respeito à discussão usual da 
interação dos efeitos acelerador e multiplicador. Dado que interpreta o modelo com contínua plena utilização da capacidade, inclusive na posição inicial, sua análise matemática diz respeito à constância (ou não) no tempo da taxa garantida de crescimento num modelo em que a cada período o investimento se ajusta à poupança potencial da economia, ou seja, lei de Say. Ao fazer isso, constata (como já vimos acima) que essa taxa garantida de crescimento equilibrado só pode se manter constante ao longo do tempo se, por acaso, ocorrer a coincidência entre a taxa garantida e a taxa de crescimento do consumo autônomo.

Ademais, como a suposição de que a taxa de crescimento dos gastos autônomos $(z)$ é inferior a $s / v$ é estrutural para o modelo do supermultiplicador sraffiano, ${ }^{11}$ esse autor conclui que a taxa garantida $\left(g_{w}\right)$ (e a própria taxa de crescimento da economia, se considerada a hipótese de contínua plena utilização da capacidade) vai tender assintoticamente a $s / v$. A ideia subjacente é de que o peso da "despoupança" causada pela existência dos gastos autônomos, $Z / Y$, tende a diminuir no tempo, aproximando a taxa garantida para $s / v$. Dessa análise, conclui que o modelo, interpretado sob crescimento equilibrado à taxa $z$, seria inerentemente instável, independentemente de valores específicos de outros parâmetros, já que $z<s / v$, e a isso atribui o acelerador rígido; algo que já não mais é nem mesmo o acelerador rígido ultraespecífico (e já muito questionável) de Trezzini, mas que se desconecta completamente do conceito de acelerador. Esse resultado formal é inteiramente baseado na reinterpretação de um modelo de crescimento liderado pela demanda como sendo liderado pela oferta. Logo, não apenas não oferece uma crítica relevante como também não apresenta propriamente uma análise de estabilidade, já que a capacidade é continuamente suposta igual à demanda.

Park (2000) chega a essas mesmas conclusões, usando o mesmo método, mas acrescenta um novo resultado: sempre que $s / v>z$ para qualquer período finito de análise, haverá uma relação inversa entre a taxa de crescimento da economia e a taxa de crescimento dos gastos autônomos, $(z)$, mesmo com a economia convergindo assintoticamente para $s / v$ A ideia é que, quanto maior o valor de $z$ para um dado valor de $s / v$, maior será o valor médio de $Z / Y$, logo, maior será a redução da poupança potencial da economia e, portanto, menores serão os valores da taxa garantida de crescimento num intervalo de tempo particular. Vê-se aí um trade-off entre consumo e investimento que, aliás, constitui o motivo pelo qual associamos explicitamente os críticos desta seção à contraditória e particular combinação de "lei de Say com acelerador rígido".

11 Detalhes acerca dessa condição constam na seção 5 . 
Esse resultado de Park (2000), a exemplo dos outros críticos vistos anteriormente, é exatamente o oposto do que postula Serrano (1995a; 1995b). Para o último, uma maior taxa de crescimento dos gastos autônomos aumentaria a taxa de crescimento do produto e da capacidade produtiva da economia.

\subsection{LEITURA 4: INSTABILIDADE DINÂMICA NO ACELERADOR RÍGIDO (SCHEFOLD, DE-JUAN E WHITE)}

Enfim, chegamos ao quarto tipo de crítica, que nos parece aquele relativamente mais coerente: a possibilidade de que um acelerador rígido na sua forma geral gere uma tendência à instabilidade dinâmica num modelo de crescimento liderado pela demanda.

Neste roteiro vamos incluir a crítica verbal de Schefold (2000), ${ }^{12}$ porém, com uma ressalva inicial. Embora ao longo do seu texto Schefold (2000) levante a questão sobre a provável instabilidade dinâmica do supermultiplicador sraffiano, que considera baseado no acelerador rígido, ocorre que sua análise formal é basicamente a mesma utilizada por Barbosa-Filho e Park, algo que nos deixa sem uma opção clara sobre qual das duas interpretações críticas melhor reflete a posição teórica do autor.

Segundo a "leitura 4" das críticas, entende-se, corretamente, o acelerador rígido associado à tentativa das firmas de ajustarem plena ou imediatamente o estoque de capital a qualquer variação de demanda, de modo que a reação do investimento se torna proporcional à relação capital-produto, $v$. Schefold acrescenta à sua crítica $o$ argumento de que os valores empíricos de $v$ já seriam muito maiores que 1 . Como isso faria a propensão a investir em desequilíbrio ser maior que 1, isso bastaria para tornar a propensão marginal a gastar da economia maior que 1 independente dos valores de outros parâmetros. Mas, nesse caso, o supermultiplicador sraffiano é sempre dinamicamente instável (como veremos na discussão das condições formais de estabilidade na seção 5 abaixo), e, portanto, inadequado para analisar a tendência de crescimento de longo prazo.

Já De-Juan $(2005,2013)$, depois de também argumentar que o acelerador utilizado em Serrano (1995) é rígido, propõe uma versão de supermultiplicador com um suposto mecanismo estável e estabilizador. Sua função investimento é determinada supondo que os investidores privados de alguma forma já sabem que a tendência da taxa de 12 Uma versão em inglês de Schefold (2000) foi apresentada em 1998 numa conferência em Roma, mas
somente a versão em italiano foi publicada no livro com os ensaios da conferência. 
crescimento da economia é dada pela taxa de crescimento dos gastos autônomos. Ademais, supõe também que os investidores ignoram outras flutuações da demanda e investem de forma a fazer o estoque de capital e a capacidade produtiva da economia sempre crescerem à taxa de crescimento esperada (aproximada pela taxa recentemente observada) dos próprios gastos autônomos, o que se parece muito pouco com qualquer tipo de acelerador. A função investimento de De-Juan é dada por:

$$
I=\Delta K=\left(\frac{v}{u}\right) z_{-1} Y
$$

Note que, nessa função, há uma correlação pouco usual entre um menor grau efetivo de utilização da capacidade e um maior nível e parcela do investimento no produto. Levando em conta que $Y / u=Y^{*}$, podemos reescrever a equação (14) como:

$$
I=v z_{-1} Y^{*}=z_{-1} K
$$

Onde vemos que o investimento depende apenas do crescimento dos gastos autônomos. No artigo de 2013, De-Juan chama sua função investimento de acelerador "flexível" e, em 2005, de "prospectivo", contrapondo-o ao "retrospectivo" que atribui ao supermultiplicador sraffiano, porque este responderia às variações realizadas e não às esperadas no futuro. Mas vemos que, em (15), a tendência da acumulação de capital é fixada diretamente, não havendo, a rigor, nenhum mecanismo acelerador. O modelo é bastante estável e converge monotonicamente ${ }^{13}$ (sem apresentar as flutuações cíclicas amortecidas típicas de modelos de ajuste do estoque de capital), mas essa estabilidade é obtida por hipótese e não advém da análise teórica do processo competitivo de ajustamento da capacidade à tendência da demanda.

White (2008a; 2008b) também considera que o supermultiplicador sraffiano é rígido, e nota que uma formulação de acelerador rígido na qual a propensão marginal a investir é função da tendência de crescimento esperada da demanda faria o

${ }^{13}$ A rigor, o modelo só gera alguma flutuação porque são admitidos erros de expectativas de curto prazo em relação à demanda corrente, algo por simplificação usualmente ignorado nos modelos acelerador multiplicador e de supermultiplicador que utilizam capital fixo. Por outro lado, o problema de ajuste do estoque de capital circulante que advém da consideração desses erros (pois há variações indesejadas de estoques), e que por si só levariam a possíveis flutuações cíclicas, é ignorado pelo autor. No entanto, dois estudos econométricos recentes, feitos em coautoria com o próprio autor (ARESTIS, GONZALEZ e DE-JUAN, 2012; GONZALEZ, ARESTIS e DE-JUAN, 2013) encontram que tanto a taxa de crescimento recente da demanda agregada como um todo (e não apenas dos gastos autônomos), quanto os desvios do grau de utilização, têm efeitos positivos e significativos sobre a taxa de acumulação de capital. 
crescimento esperado ser igual à taxa de crescimento observada no período mais recente. A partir daí, argumenta que as propensões marginais a investir e a gastar tendem a ficar muito altas, tornando o modelo dinamicamente instável. Para mudar a determinação da propensão marginal a investir do supermultiplicador, o autor propõe uma formulação diferente para a taxa de crescimento esperada da demanda, formada por uma média ponderada de dois componentes: um deles é a taxa de crescimento da demanda autônoma observada no período anterior, $z$; o outro é uma média da taxa de crescimento da demanda como um todo nos dois períodos anteriores, com um fator de desconto para a dispersão entre essas duas taxas. Formalmente:

$$
g^{e}=a\left[\frac{g_{-1}+g_{-2}}{2}\right]\left[\frac{1}{1+b\left(g_{-1}-g_{-2}\right)}\right]+(1-a) z
$$

White mostra que, quanto maior o peso $(1-a)$ da demanda autônoma $(z)$ nas expectativas, e quanto maior o fator de desconto $(b)$ para a dispersão entre as duas taxas de crescimento da demanda agregada mais recentes, maior é a chance de o modelo ser dinamicamente estável. A linha de pesquisa de White, com o uso frequente de simulações de computador, sobre as implicações para a estabilidade dinâmica do supermultiplicador sraffiano de diferentes formas de expectativas adaptativas e de aprendizado, parece-nos bastante promissora.

Por outro lado, como discutido sobre De-Juan $(2005,2013)$, embora seja claro que a formulação do acelerador rígido tenda a gerar instabilidade dinâmica, não parece razoável supor que os investidores, ou mesmo uma fração deles, esperem que a economia vá crescer de fato à taxa que crescem os gastos autônomos. Além disso, o segundo componente de White (2008a, 2008b) nos parece ainda próximo demais ao acelerador rígido, pois implica que as empresas que formulam expectativas dessa forma tentam ajustar plenamente a capacidade à demanda observada em apenas dois períodos. Esta, inclusive, pode ser a razão pela qual White chega ao resultado de que o parâmetro de desconto deve tomar valores bem altos para amortecer as mudanças na propensão marginal a investir e permitir a estabilidade dinâmica do modelo.

\section{O SUPERMULTIPLICADOR SRAFFIANO COM ACELERADOR FLEXÍVEL}

Na seção 3, vimos que o ajustamento da taxa de crescimento esperada em direção à taxa de crescimento dos gastos autônomos que faria o supermultiplicador sraffiano tender à sua posição de pleno ajustamento, não foi orginalmente demonstrada a contento. Uma versão completa do modelo, com acelerador flexível, cumpre esse papel. 
A função investimento com acelerador rígido, essencialmente descrita na literatura keynesiana por versões equivalentes a (6), sofreu, em síntese, duas críticas centrais. Teoricamente, questionou-se a consistência de supor que, se inicialmente houvesse capacidade ociosa não planejada, as empresas investiriam exatamente em proporção ao aumento da demanda, já que tal volume de investimentos não seria estritamente necessário. Empiricamente, praticamente todos os estudos desde os anos 1930 até os dias de hoje demonstraram que o nível de investimento sempre reage significativamente às flutuações de demanda, porém com muito menos intensidade do que o previsto pelo modelo do acelerador rígido (MATTHEWS, 1959).

Tais críticas levaram ao uso generalizado do chamado acelerador flexível ou princípio do ajuste do estoque de capital, em que se supõe que as empresas em geral sabem que a demanda, o produto e o grau efetivo de utilização da capacidade sofrem flutuações temporárias, que não são indicadores dos aumentos futuros da tendência da demanda. Adicionalmente, no caso de investimento em capital fixo, a própria durabilidade do equipamento por vários períodos de produção implica que as empresas não desejam obter a utilização normal da capacidade a cada período e sim somente na média da vida útil do equipamento.

Por conta dessas considerações, o modelo do acelerador flexível aparece em duas formas alternativas, mas que são equivalentes. Ou se usam expectativas adaptativas com ajuste parcial e defasagens distribuídas em que as expectativas são revisadas gradualmente à luz da experiência ou então o investimento é determinado por um processo de ajuste gradual ou parcial do estoque de capital corrente ao estoque de capital desejado captado pelas discrepâncias entre o grau efetivo e o planejado de utilização de capacidade. Em ambos os casos (e é possível combiná-los), o investimento reage bem menos às flutuações da demanda do que no caso do acelerador rígido, algo que aumenta em muito o realismo do modelo.

Especificações do supermultiplicador a partir de mecanismos de ajustamento de acelerador flexível como os descritos acima são mais realistas pois se baseiam num tipo de expectativa adaptativa na qual o processo de ajustamento se dá por tentativa e erro, não sendo necessário supor que as firmas conheçam a estrutura e o funcionamento agregado da economia. Isso evita hipóteses como as empresas saberem que são os gastos autônomos que lideram o crescimento da economia (como em White e DeJuan), ou conhecerem e distinguirem a priori as variações temporárias das variações permanentes da demanda (como em De-Juan). Versões do supermultiplicador sraffiano com acelerador flexível, na qual a taxa de investimento varia a partir dos desvios do grau de utilização em relação ao nível normal, encontram-se em Freitas e Serrano (2015) e Serrano e Freitas (2016). Outras versões nas quais a taxa de investimento varia a partir da revisão gradual da taxa de crescimento esperada da 
tendência da demanda se encontram em Cesaratto et al. (2003), Serrano (2006), Pariboni (2015) e Serrano et al. (2018). Vamos ilustrar uma variante dessas últimas com base no que vimos na seção 3, sobretudo no conteúdo da equação 7, explorando agora de que forma será feita a revisão de expectativas. Suporemos que $g^{e}$ é corrigida gradualmente à luz dos resultados realmente observados em termos de expectativas adaptativas da forma:

$$
g^{e}=g_{-1}^{e}+x\left(g_{-1}-g_{-1}^{e}\right) \text { ou } g^{e}=x g_{-1}+(1-x) g_{-1}^{e}
$$

Note que, supondo $x<1$, temos o equivalente ao acelerador flexível. E esse parâmetro deve ser suficientemente pequeno para que na vizinhança da posição de pleno ajustamento, a propensão marginal a gastar da economia se mantenha menor que 1. Por outro lado, se for suposto que $x=1$, obtemos o acelerador rígido (ver seção 2). Na hipótese de acelerador flexível, a taxa de investimento induzido fica determinada por:

$$
\frac{I}{Y}=v g^{e}=x v g_{-1}+(1-x) v g_{-1}^{e}
$$

A partir do ajuste do investimento como em (18), podemos esclarecer dois aspectos que são suficientes para determinar a "condição necessária no 3" da seção 3, conforme pretendíamos. O primeiro aspecto é de corte estrutural (ou de "equilíbrio") e já estava contemplado em Serrano (1995a). Refere-se ao fato de que, nas próprias posições de pleno ajustamento da capacidade onde o grau de utilização é o normal e a taxa de crescimento da economia é dada pela taxa de crescimento dos gastos autônomos, $z$, a propensão marginal a gastar deve ser menor que 1 . Isso significa que a taxa de crescimento dos gastos autônomos deve ser estritamente menor que a taxa máxima de crescimento da economia compatível com um regime de crescimento liderado pela demanda. Esta, por sua vez, é a taxa que induziria uma taxa requerida de investimento induzido $v . z$, que, somada à propensão marginal a consumir, geraria uma propensão marginal a gastar igual a 1. Logo, o modelo requer estruturalmente:

$$
z<(1-c) / v
$$

O segundo aspecto diz respeito ao ajustamento cíclico (ou de "desequilíbrio") e não aparece na versão original do modelo. Ocorre que, além desse limite estrutural em (19), nas versões mais recentes do modelo, que explicitamente assumem a hipótese de acelerador flexível, ficou claro que a estabilidade dinâmica requer um limite adicional: se o processo de ajustamento for especificado de forma linear como na equação (17), a propensão marginal a gastar da economia também não pode ficar acima de 1 durante 
o processo de ajustamento, enquanto o grau de utilização estiver diferente do normal e/ou a taxa de crescimento esperada for diferente da taxa de crescimento dos gastos autônomos. Porém, nesse processo de ajustamento, a propensão marginal a investir da economia tenderá a ser maior do que seu valor estrutural, $v z$, por conta da taxa de investimentos adicionais induzidos $(v x)$ por taxas de crescimento esperadas superiores a taxa de tendência, $z$ (e um fator de interação entre a taxa de tendência e ciclo $v x z$ ). Assim, na vizinhança da posição de pleno ajustamento, a propensão marginal a gastar do modelo completo com acelerador flexível seria dada por:

$$
c+v z+v x+v x z
$$

de onde podemos ver que a taxa máxima de crescimento dos gastos autônomos compatível com a estabilidade dinâmica do modelo será menor do que a da equação (19), o que implica que a taxa máxima de crescimento dinamicamente estável que mantém o modelo liderado pela demanda será dada por: ${ }^{14}$

$$
z<\frac{\left[\frac{(1-c)}{v}-x\right]}{(1+x)}
$$

e será tão menor quanto maior for o coeficiente de reação, $x$. A desigualdade (21) é suficiente para garantir plenamente a "condição necessária no 3", de modo que a própria demanda induzida gerada pelo ajustamento da capacidade à demanda não seja excessiva. ${ }^{15}$

Os limites dados por (19) e (21) nos permitem discutir uma última crítica apresentada por Trezzini $(1995,1998)$, que alega que a demanda autônoma não estaria "livre" para crescer a qualquer taxa, supostamente contradizendo a liderança do crescimento pela demanda. Essa crítica também carece de fundamento. É evidente que a demanda autônoma pode, sim, crescer a qualquer taxa. O problema é que, se o

${ }^{14}$ Para a prova formal dessa condição de estabilidade dinâmica em tempo discreto, ver Serrano, Freitas e Bhering (2018). Versões dessas provas em tempo contínuo são encontradas em Freitas e Serrano (2015), Allain (2015) e Pariboni (2015).

15 A condição (21) é suficiente, mas não estritamente necessária, e pode ser relaxada num modelo com parâmetros que variam durante o ajustamento cíclico se, por exemplo, a propensão marginal a gastar for maior que um na vizinhança da posição de pleno ajustamento, mas se tornar menor que um quando o grau de utilização se tornar muito maior que o normal. Porém, a condição estrutural (19) não pode ser relaxada, seja qual for a natureza do processo de ajustamento, e é estritamente necessária. 
crescimento da demanda autônoma for maior do que a taxa máxima, a oferta e a produção não terão como responder. Note que, se a taxa máxima de crescimento for excedida, será gerado tanto investimento induzido que a propensão marginal a gastar da economia ficará maior do que 1 , o que significa que o nível de demanda efetiva agregado tenderia ao infinito. Dado isso, fica claro que (como bem coloca De-Juan, 2005), em vez de evidenciar uma contradição lógica, a taxa máxima de crescimento é um conceito que evita a séria contradição lógica de postular que o produto pode crescer liderado pela demanda quando a demanda agregada é infinitamente grande.

\section{OBSERVAÇÕES FINAIS}

Nosso principal objetivo ao longo deste trabalho foi esclarecer a relação entre o conceito de acelerador e o modelo do supermultiplicador sraffiano e, a partir disso, resenhar os termos da controvérsia que emergiu em torno desse modelo. Como vimos, as principais críticas estavam ligadas à suposição de que o modelo estaria necessariamente associado a um conceito de acelerador rígido, seja implicando que a capacidade sempre está plena e continuamente ajustada à demanda, desde o período inicial - o que tornaria o modelo incompatível com a noção de crescimento liderado pela demanda -, seja no sentido mais usual de que, sob a hipótese de acelerador rígido, o investimento tenderia a reagir plenamente a qualquer flutuação de demanda -, o que tornaria o modelo altamente suscetível a padecer de instabilidade. Mostramos que essas duas linhas de crítica não se sustentam e que o modelo do supermultiplicador sraffiano é consistente com a noção de crescimento liderado pela demanda e também plenamente compatível com a hipótese de acelerador flexível, possibilitando sua estabilidade dinâmica. Finalmente, contrastado com as versões alternativas do supermultiplicador de De-Juan e White, constatamos que as condições para a estabilidade dinâmica da versão mais completa do supermultiplicador sraffiano prescindem de hipóteses irrealistas sobre as empresas conhecerem a priori a relevância macroeconômica e a própria magnitude da taxa de crescimento dos gastos autônomos.

Contudo, a exposição da controvérsia juntamente aos desenvolvimentos mais recentes do modelo permitiram maior clarificação conceitual ${ }^{16}$ das hipóteses suficientes para atender à terceira das condições necessárias que listamos para garantir o

16 Trezzini e Palumbo (2016, p. 511 e nota 15) mencionam que Freitas e Serrano (2015) esclareceram que as críticas de Trezzini $(1995,1998)$ só se aplicariam "se o supermultiplicador fosse interpretado" com contínua plena utilização da capacidade. 
ajustamento da capacidade à demanda. Vimos que foi a não formalização explícita dessa condição em Serrano (1995a) a origem mais provável da controvérsia. Os esforços de pesquisa teórica a partir de agora, a nosso ver, deveriam se concentrar primordialmente na análise teórica e empírica da natureza e dos diferentes determinantes, particularmente financeiros, dos diversos tipos de gastos autônomos que não criam capacidade (consumo financiado por crédito, investimento residencial, exportações, gastos públicos), por muito tempo negligenciados na literatura heterodoxa de modelos de crescimento. ${ }^{17}$

\section{REFERÊNCIAS}

ACKLEY, G. Un modello econometrico dello sviluppo italiano nel dopoguerra. Milano: Giuffrè, 1963. Disponível em: <www.svimez.info/images/pubblicazioni/monografie/m_19.pdf>.

ALLAIN, O. Tackling the instability of growth: a Kaleckian-Harrodian model with an autonomous expenditure component. Cambridge Journal of Economics, v. 39, n. 5, 2015.

ARESTIS, P.; GONZALEZ, A.; DE-JUAN, O. Private productive investment in Spain and the United States. Análise Econômica, v. 30, n. 58. p.7-27, 2012.

BARBOSA FILHO, N. A note on the theory of demand-led growth. Contributions to political economy, v. 19, n. 1, p. 19-32, 2000.

BORTIS, H. Foreign resources and economic development from the early fifties to the oil crisis: a consideration of some theoretical and empirical aspects. Suiça: Editions Universitaires Fribourg, 1979.

BROCHIER, L.; MACEDO E SILVA, A. C. The macroeconomic implications of consumption: state-of-art and prospects for the heterodox future research. In: ENCONTRO NACIONAL DE ECONOMIA POLÍTICA, 20, Foz do Iguaçu, Paraná, dez. 2015.

CESARATTO, S. Neo-Kaleckian and Sraffian controversies on the theory of accumulation. Review of Political Economy, v. 27, n. 3, p. 154-182, 2015.

CESRATTO, S. Garegnani, Ackley and the years of high theory. Centro Sraffa Working Papers, n. 26, nov. 2017.

CESARATTO, S.; SERRANO, F.; STIRATI, A. Technical change, effective demand and employment. Review of Political Economy, v. 15, p. 33-52, 2003.

CICCONE, R. Accumulation and capacity utilization: some critical considerations on Joan Robinson's theory of distribution. Political Economy: Studies in the Surplus Approach. v. 2, p. 17-36.

${ }_{17}$ Brochier e Macedo e Silva (2015), Fibieger e Lavoie (2017) e Cesaratto (2016) apresentam conclusões semelhantes. 
DE-JUAN, O. Paths of accumulation and growth: towards a Keynesian long-period theory of output. Review of Political Economy, v. 17, n. 2, p. 231-252, 2005.

DE-JUAN, O. "Normal paths of growth shaped by the supermultiplier". In: LEVRERO, E.; PALUMBO, A.; STIRATI, A. (Orgs.) Sraffa and the reconstruction of economic theory. Vol. 2. Basingstoke: Palgrave Macmillan, 2013.

FIEBIGER B.; LAVOIE, M. Trend and business cycles with external markets? Non-capacity generating semi-autonomous expenditures and effective demand. Metroeconomica, forthcoming, 2017.

FREITAS, F.; SERRANO, F. Growth rate and level effects, the adjustment of capacity to demand and the Sraffian supermultiplier. Review of Political Economy, v. 27, n. 3, p. 258-281, 2015.

GAREGNANI, P. "Some notes for an analysis of accumulation". In: HALEVI, J.; LAIBMAN, D.; NELL, E. J. (Eds). Beyond the steady state: a revival of growth theory. New York: St Martin's Press, 1992.

GAREGNANI, P. The problem of effective demand in Italian economic development: on the factors that determine the volume of investment. Review of Political Economy, v. 27, n. 2, p. 111-133, 1962[2015].

GARRIDO MOREIRA, V. Demanda Efetiva e Crescimento na Abordagem Sraffiana: fundamentos teóricos e aplicações. Dissertação (Mestrado em Economia) - Instituto de Economia, Universidade Federal do Rio de Janeiro, Rio de Janeiro, RJ, Brasil, 2007.

GARRIDO MOREIRA,V.; SERRANO, F. Demanda efetiva no longo prazo e no processo de acumulação: origem e desenvolvimento do debate sraffiano a partir do projeto de Garegnani (1962). Economia e Sociedade, v. 27, n. 2, p. 463-492, mai./ago., 2018.

GONZALEZ, A.; ARESTIS, P.; DE-JUAN, O. "Business investment, growth and crisis". In: DEJUAN, O.; FEBRERO, E.; UXÓ, J. (Eds.). Post-Keynesian views of the economic crisis and its remedies. London; New York: Routledge, 2013.

HICKS, J. A contribution to the theory of the trade cycle. Oxford: Clarendon, 1950.

LAVOIE, M. Post-Keynesian economics: new foundations. Aldershot, UK and Brookfield, VT: Edward Elgar, 2014.

LAVOIE, M. Convergence towards the normal rate of capacity utilization in Neo-Kaleckian models: the role of non-capacity creating autonomous expenditures. Metroeconomica, v. 67, n. 1, p. 172-201, 2016.

MATTHEWS, R. C. O. The trade cycle. Cambridge: James Nisbet, 1959.

PALUMBO, A. "Potential output and demand-led growth. In: LEVRERO, E.; PALUMBO, A.; STIRATI, A. (Orgs.) Sraffa and the reconstruction of economic theory. v. 2 Basingstoke, UK: Palgrave Macmillan, 2013.

PARK, M. S. Autonomous demand and the warranted rate of growth. Contributions to Political Economy, v. 19, p.1-18, 2000.

SCHEFOLD, B. “Fasi dell'accumulazione e mutevoli influenze sulla distribuizione”. In: PIVETTI, M. (Ed.). Piero Sraffa: contributti per una biografia intellettuale. Rome: Carocci, 1998[2000].

SERRANO, F. Long period effective demand and the Sraffian supermultiplier. Contributions to Political Economy, v. 14, p.67-90, 1995a. 
SERRANO, F. The Sraffian supermultiplier. Tese (Doutorado em Economia) - Faculty of Economics and Politics, University of Cambridge, Cambridge, UK, United Kingdom, 1995b.

SERRANO, F. "Acumulação e gasto improdutivo na economia do desenvolvimento". In: FIORI, J. L.; MEDEIROS, C. (Org.). Polarização mundial e crescimento. Petrópolis: Vozes, 2001.

SERRANO, F. Mind the gap: hysteresis, inflation dynamics and the Sraffian supermultiplier. Rio de Janeiro: IE-UFRJ, 2006. (mimeo).

SERRANO, F.; FREITAS, F. The Sraffian supermultiplier as an alternative closure for heterodox growth theory. European Journal of Economics and Economic Policies Intervention, v. 14, n. 170-191, 2016.

SERRANO, F.; FREITAS, F.; BHERING, G. The trouble with Harrod: the fundamental instability of the warranted rate in the light of the Sraffian Supermultiplier. Metroeconomica, v. 70, n. 2, oct. 2018.

PALUMBO, A.; TREZZINI, A. Growth without normal capacity utilization. European Journal of the History of Economic Thought, v. 10, n. 1, p. 109-135, 2003.

PARIBONI, R. Autonomous demand and capital accumulation: three essays on heterodox growth theory. Dissertação (Ph.D. em Economia) - Departamento de Economia Política e Estatística, Universidade de Siena, 2015.

TREZZINI, A. Capacity utilisation in the long run and the autonomous components of aggregate demand. Contributions to Political Economy, v. 14, p. 33-66, 1995.

TREZZINI, A. Capacity utilisation in the long run: some further considerations, Contributions to Political Economy, v. 17, p. 53-67, 1998.

TREZZINI, A. "The meaning of output trends in the analysis of growth". In: LEVRERO, E.; PALUMBO, A.; STIRATI, A. (Orgs.) Sraffa and the reconstruction of economic theory. v. 2. Basingstoke, UK: Palgrave Macmillan, 2013.

TREZZINI, A.; PALUMBO, A. The theory of output in the modern classical approach: main principles and controversial issues. Review of Keynesian Economics, v. 4, p. 503-522, 2016.

WHITE, G. Growth, autonomous demand and a joint product treatment of fixed capital. Metroeconomica, v. 59, n. 1, p. 1-26, 2008a.

WHITE, G. "Demand-led growth and the classical/Sraffian approach to value and distribution - are they compatible?" In: SALVADORI, N. (Ed.). Economic growth and distribution: on the nature and causes of the wealth of nation. Cheltenham: E. Elgar, 2008b. 